\title{
Analysis of Complementary Characteristics of Wind/PV/hydro Power Based on the Bundled Output
}

\author{
Yanhong Dou ${ }^{1}$, Wei Ding ${ }^{1, a}$, Yan Huang ${ }^{2}$, Jing $\mathrm{Hu}^{1}, \mathrm{Yu} \mathrm{Li}^{1}$ and Huicheng Zhou ${ }^{1}$ \\ ${ }^{1}$ School of Hydraulic Engineering, Dalian University of Technology, Dalian 116024, China. \\ ${ }^{2}$ Yalong River Hydropower Development Company, Ltd, Chengdu, 610056, China.
}

\begin{abstract}
There is complementarity among wind, photovoltaic, and hydro power (wind/PV/hydro power), which is closely related to the bundled installed capacity of wind/PV/hydro complementary power generation system and regulating ability of hydropower. This paper evaluates the complementary characteristics among wind, PV, and hydro power though the ability of the system to output power smoothly. First, the ratio of installed capacity of wind to PV power is determined by minimizing the volatility of wind/PV joint power generation. Second, an optimization model, with the objective of maximizing grid-connected and steady output of wind/PV/hydro complementary system in the dry season, to determine the bundled output of the system is proposed. Third, the complementary characteristics of wind/PV/hydro power are analyzed by the bundled output and the absorptive rate of new energy in the dry season. The implication in the Yalong River shows that: (1) The optimum ratio of installed capacity of wind to PV power is 0.7 . (2) The bundled output of wind/PV/hydro system is $1.742 \mathrm{GW}$, in which $6.3 \mathrm{GW}$ are bundled capacity of wind/PV/hydro power. (3) The complementarity of wind/PV/hydro power will be strengthened with the increase of runoff in the dry season and the decrease of the new energy installed capacity. The research can provide guidelines for the planning and design of the clean energy complementary base.
\end{abstract}

\section{Introduction}

The exploitation of wind/photovoltaic/hydro (wind/PV/hydro) complementary system in a basin is of great significance to improve the utilization of clean energy and optimize the power grid structure [1]. The wind and PV power are random, volatile and intermittent $[2,3]$, but also complementary [4] due to climate and meteorological factors. Hydropower can smooth the volatility of wind and PV power through the regulation of reservoir, which regulates the inflow variability. Therefore, the primary task of the exploitation of the wind/PV/hydro complementary power generation system is to analysis the complementary characteristics among wind, PV and hydro power.

Wind/PV/hydro complementary power generation system is one of the hybrid renewable energy generation systems (HREGS), which also includes systems of wind/hydro energy, PV/hydro energy and wind/PV energy, etc. [4]. The analysis methods for the complementary characteristics of different HREGS are similar, and there are two types: non-grid-connected and grid-connected. To smooth the output fluctuations of HREGS, the former studies tend to use the regulating and storing function of reservoir, while the latter studies tend to use energy storage device. In existing research, for the grid-connected HREGS, researchers analyzed the seasonal and intraday relationship between peak and valley of variation curves

\footnotetext{
a Corresponding author: weiding@dlut.edu.cn
}

of three kinds of energy to discuss the complementarity qualitatively, based on the historical data of independent output of wind, PV and hydro power. For instance, X. Zhang et al. [4] and X. Ye et al. [8] both analyzed the annual and intraday complementary characteristics among wind power, PV power and independent hydropower. X. Zhang et al. [4] considered the impact of different weather conditions on the daily complementary characteristics. But none of the above-mentioned studies have analyzed the complementary characteristics from the view of multienergy bundle output and taking into account the regulating and storing function of reservoir concurrently. For the non-grid-connected HREGS, wind power, PV power and micro hydropower without the performance of regulation are bundled to generate power, and then controlled by the energy storage device to independently balance the generation and the small load. Therefore, researchers analyzed complementarity by the theoretical storage required and the indicators of power balance quantitatively. For instance, Khan et al. [6] described the complementarity of stand-alone solar/wind hybrid energy system by hydrogen used as an energy transporter, and R.K. Akikur [7] described it by the requirement of the storage battery. D. Raynaud et al. [1] evaluated the complementarity of wind/PV/hydro energy with the indicators of energy production droughts and energy supply droughts. François, B. et al. [9] analyzed the complementarity with the standard deviation of the energy balance. By comparing various indicators, researchers 
found out that it is more reliable to use a hybrid power generation system than a single photovoltaic power generation system or a wind power generation system $[10$, 11]. It can be seen that the quantitative method is more conducive to the evaluation and comparison of the complementary characteristics, and its idea can be borrowed to the grid-connected HREGS. However, for the grid-connected HREGS, there is the dual function of hydropower: power generation and energy storage, while the hydropower of non-grid-connected HREGS can only generate power.

Therefore, this paper proposed to evaluate the complementary characteristics of the wind/PV/hydro complementary power generation system by using the ability of the system to output the bundled power smoothly on a large scale, which is affected by the exploitation scale of wind, PV, and the regulation ability of the hydropower. First, the wind speed and solar irradiance are transformed into the output coefficients of wind and PV power, respectively. Based on the analysis of wind and PV power characteristics, combined with the variation coefficients, the optimal ratio of the installed capacity of wind to PV power is obtained by minimizing the joint output volatility of wind/PV power. Second, an optimization model, maximizing the grid-connected and steady output of wind/PV/hydro complementary power generation system in the dry season through the allocation of hydropower generation, to determine the bundled output is proposed. Following that, the impact of the bundled wind/PV/hydro power on the bundled output are analyzed. Finally, the complementary characteristics of wind/PV/hydro complementary power generation system in Yalong River are analyzed.

\section{Bundled output optimization model of wind/PV/hydro complementary system}

The complementary characteristics of wind/PV/hydro energy are reflected in the ability of hydropower to regulate the wind/PV power to output the power smoothly and massively. To reduce the burden of hydropower to regulate the output of wind and PV power, it is necessary to determine the optimal ratio of installed capacity of wind to PV power to minimize the volatility of the joint power output of wind/PV power. The regulation ability of hydropower in the dry season is limited by the reservoir capacity and inflow. The purpose of this paper is to analyze the complementary characteristics in the planning stage, therefore, the calculation of total hydropower generation in the dry season is simplified, and the power generation is reallocated to smooth the volatility of wind/PV and further to maximize the bundled output of the system. The bundled output of the wind/PV/hydro complementary system $\left(N_{b}\right)$ is defined as the gridconnected and steady output of the wind/PV/hydro complementary power generation system in the dry season.

\subsection{Objective function}

The objective of the model is to maximize the minimum bundled output of the wind/PV/hydro power by the regulation of hydropower.

$$
\max N_{b}=\max \left[\min \left(N_{H, t}+N_{W P V, t}\right)\right]
$$

where $N_{H, t}$ denotes the hydropower output in the $t$ th period; $N_{W P V, t}$ denotes the wind/PV power output in the $t$ th period, determined by the product of the installed capacity of wind/PV power and the output coefficients of wind/PV power:

$$
N_{W P V, t}=N_{r, b} \cdot \eta_{W P V, t}\left(m_{o p}\right)
$$

where $N_{r, b}$ denotes the installed capacity of wind/PV power; $\eta_{W P V, t}\left(m_{o p}\right)$ denotes the output coefficients of wind/PV power with the optimal installed capacity ratio of $m_{o p}$ in the $t$ th period:

$$
\eta_{W P V, t}\left(m_{o p}\right)=\frac{\eta_{P V, t}+m_{o p} \eta_{W, t}}{1+m_{o p}}
$$

where $\eta_{W, t}$ denotes the output coefficients of wind power in the $t$ th period; $\eta_{P V, t}$ denotes the output coefficients of PV power in the $t$ th period; $m_{o p}$ denotes the optimal installed capacity ratio of wind to PV power which minimizes the output volatility of wind/PV joint generation.

\subsubsection{Calculation of output coefficients of wind and PV power}

The output coefficients of wind and PV power are defined as the output of one unit of installed capacity, expressed as follows:

$$
\eta_{t}=N_{t} / N_{r}
$$

where $N_{t}$ denotes the output in period t, and $N_{r}$ denotes the installed capacity. The wind speed sequence that can reflect the wind energy and the solar intensity sequence that can reflect the solar energy are converted into the output coefficients sequences. The output coefficient of wind power in period $t$ can be calculated with the quadratic model [12]:

$$
\eta_{W, t}=\left\{\begin{array}{cc}
0 & 0 \leq v_{t} \leq v_{c i} \\
A+B v+C v^{2} & v_{c i} \leq v_{t} \leq v_{r} \\
1 & v_{r} \leq v_{t} \leq v_{c o} \\
0 & v_{t} \geq v_{c o}
\end{array}\right.
$$

where $A, B, C$ are the parameters of the characteristic curve of the wind turbine; $v_{t}$ denotes the average wind speed at the wind turbine hub in period $t ; v_{c o}$ denotes the cut-out wind speed of wind turbine; $v_{c i}$ denotes the cut-in wind speed of wind turbine; $v_{r}$ denotes the rated wind speed of wind turbine.

The output coefficient of PV power in period $t$ is computed as [13]:

$$
\eta_{P V, t}=\frac{I_{t}}{I_{s t c}}\left[1+\alpha_{T}\left(T_{c e l l, t}-T_{s t c}\right)\right]
$$

where $I_{s t c}$ denotes the irradiance of standard conditions $\left(1000 \mathrm{~W} / \mathrm{m}^{2}\right) ; T_{\text {stc }}$ denotes the temperature of standard conditions $\left(25^{\circ} \mathrm{C}\right)$; $I_{t}$ denotes the actual irradiance in period $\mathrm{t} ; \alpha_{T}$ denotes the temperature coefficient of the PV panels(-0.4\% -0.45\%); $T_{t}^{\text {cell }}$ denotes the temperature of the PV panels in period t. Due to the fact that there are only solar intensity and temperature data of the station during the planning stage of PV power plants, it is necessary to convert the temperature of air to the 
temperature of the PV panels. And the conversion formula is expressed as follow:

$$
T_{c e l l, t}=T_{a i r, t}+\frac{T_{N O C}-T_{s t c}}{I_{s t c}} I_{t}
$$

where $T_{N O C}$ denotes the normal operating cell temperature of $\mathrm{PV}$ power $\left(48 \pm 2{ }^{\circ} \mathrm{C}\right)$.

\subsubsection{The optimal installed capacity ratio of wind to PV power}

The volatility of the joint output of wind and PV power are affected by their installed capacity ratio. In order to maximize the bundled output of the system, the optimal installed capacity ratio $\left(m_{o p}\right)$ of wind to PV power is determined with the goal of minimizing the output volatility of wind/PV joint generation,

$$
m_{o p}=\operatorname{argmin} C_{v}(m)
$$

In which, the installed capacity ratio $(\mathrm{m})$ of wind to PV power is computed as

$$
m=\frac{N_{r, W}}{N_{r, P V}}
$$

where $N_{r, W}$ is the installed capacity of wind power; $N_{r, P V}$ is the installed capacity of PV power.

The coefficient of variation, measuring the output volatility of wind/PV joint generation is calculated by the following equation,

$$
C_{v}(m)=\sqrt{\frac{\sum_{t=1}^{n}\left(\frac{\eta_{W P V, t}(m)}{\bar{\eta}_{W P V, t}(m)}-1\right)^{2}}{n}}
$$

where $n$ is the length of time series.

\subsection{Constraints}

(1)Maximum grid- connected hydropower output

The total grid-connected output of hydropower can not exceed the total hydropower output,

$$
\sum_{t=1}^{n} N_{H, t} \Delta t \leq K\left(W_{d r y}+V\right) \bar{H}
$$

The right side of the inequality represents the total hydropower generation in the dry season, while the left side represents the total grid-connected hydropower output. $\Delta t$ is the account step; $K$ is the output coefficient of hydropower; $W_{d r y}$ is the total runoff in the dry season; $V$ is the usable storage; $\bar{H}$ is the average water head.

The average water head in the dry season is computed as:

$$
\bar{H}=\bar{Z}_{u}-\bar{Z}_{d}
$$

where $\bar{Z}_{u}$ and $\bar{Z}_{d}$ are the average water level of upstream and downstream, respectively; For the annual or seasonal regulation reservoir, $\bar{Z}_{u}$ is simplified to the water level corresponding to the average impoundage in the dry season $(\bar{V})$ of the reservoir, and $\bar{V}$ is estimated by the formula(13).

$$
\bar{V}=V_{\text {dead }}+V / 2
$$

(2)Minimum hydropower output constraints

The reservoir has to satisfy the minimum discharge for downstream ecology, navigation and irrigation, etc., represented by $Q_{\min }$, that is, the hydropower generation of each period should satisfy:

$$
\begin{aligned}
N_{H, t} & \geq N_{H, \text { min }} \\
N_{H, \text { min }} & =K Q_{\text {min }} \bar{H}
\end{aligned}
$$

where $N_{H, \min }$ is the minimum hydropower output.

(3)Output of wind/PV power constraints

The grid-connected output of wind/PV power cannot exceed the output of wind/PV joint generation.

$$
N_{W P V, t}^{c} \leq N_{W P V, t}
$$

Meanwhile, because the minimum output of hydropower must be ensured, the grid-connected output of wind/PV power cannot exceed the maximum allowable grid-connected output.

$$
N_{W P V, t}^{c} \leq N_{b}-N_{H, \min }
$$

Due to the strong volatility of the wind and PV output and the limited regulating ability of hydropower, the output of wind/PV power may not be absorbed fully. The absorptive rate of wind/PV power in the dry season is computed as:

$$
r_{a}=\sum_{t=1}^{n} N_{W P V, t}^{c} / \sum_{t=1}^{n} N_{W P V, t}
$$

\section{Case study}

\subsection{Overview}

Yalong River, ranking the third largest hydropower base in China, has the hydropower installed capacity of $30 \mathrm{GW}$. Meanwhile, Yalong River has rich wind, solar energy, and the planning installed capacity of wind/PV power is 30.69 GW. Therefore, a seasonal hydropower station of Yalong River and the planned wind and PV power station in the vicinity of the hydropower station have been selected as the research objects in this paper. The dry season of the basin is from November to May in the next year. The hydropower station has a maximum conservation storage of $3.37 \times 10^{9} \mathrm{~m}^{3}$, a dead storage of $2.42 \times 10^{9} \mathrm{~m}^{3}$, and an installed capacity of $3.3 \mathrm{GW}$. The variation of annual runoff is shown in Fig. 1, in which the runoff above the upper dotted line represents the wet year, and that below the lower dotted line represents the dry year.

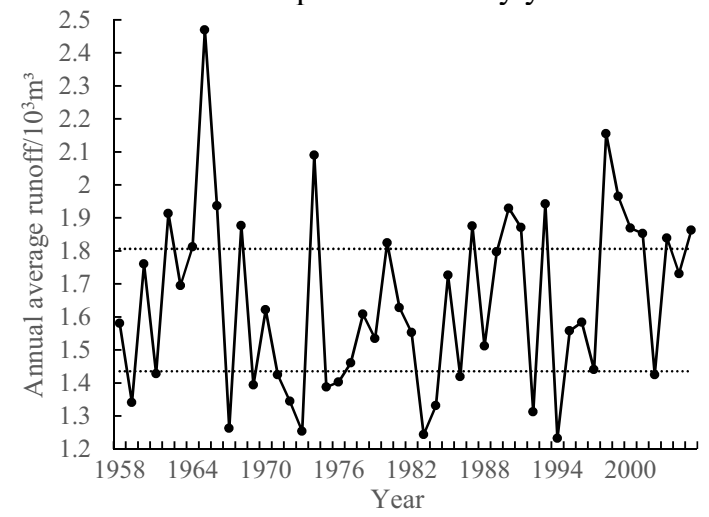

Fig. 1. Variation of annual runoff

Using the software of Meteonorm7.2, the 1-hour sequences of wind speed at $70 \mathrm{~m}$-height and the solar intensity at the optimal tilt of PV panels from 2007 to 2016 are obtained. In general, the optimal tilt angle of PV panels is the latitude plus $0^{\circ} \sim 2^{\circ}$. Results show that the inter- 
annual variation of wind speed and solar intensity is small [14], therefore, Fig. 2 shows the variations of wind speed and solar intensity in 2016.



(a)



(b)

Fig. 2. Variations of wind speed (a) and solar intensity (b) in 2016

\subsection{Complementarity analysis of wind and PV power}

Assuming that the cut-in wind speed of the wind turbine is $3 \mathrm{~m} / \mathrm{s}$, the rated wind speed is $10 \mathrm{~m} / \mathrm{s}$, and the cut-out wind speed is $20 \mathrm{~m} / \mathrm{s}$. Then, the 1 -hour sequences of wind speed and solar intensity from 2007 to 2016 are transformed into the output coefficients of wind and PV power, respectively. These data are classified into hourly collections of each month. There are 24 collections per month, and 288 collections in 12 months of one year. The variations of the average output coefficients of wind and PV power in each hour of different months throughout the year are shown in Fig. 3 by the full line and the imaginary line, in which the circle and diamond dots denote the average monthly output of wind and PV power, respectively. The shadow parts of the figure show the intraday variations of wind and PV power output in April and September, respectively.

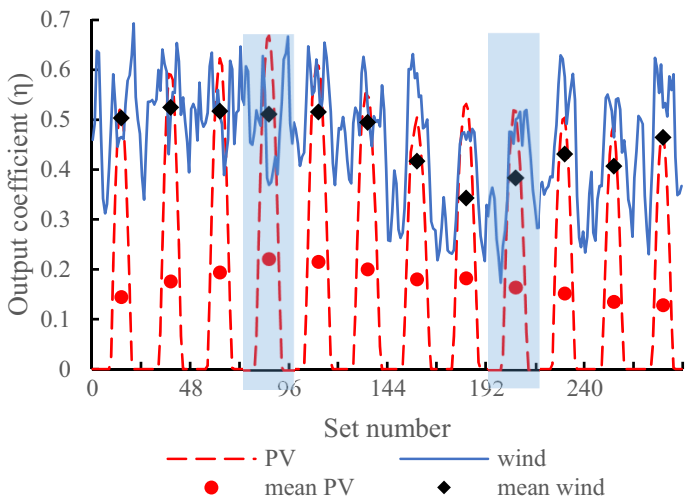

Fig. 3. Variations of average output coefficients of wind and $\mathrm{PV}$ power

The seasonal and daily characteristic of wind and PV power can be analyzed in Figure 3. Seasonally, wind power generation is abundant in the first half of the year, but deficient in the second half of the year. Daily, the regularity of intraday variation varies from month to month. For instance, the fluctuations are frequent but the amplitude is small in the first half of the year, while the amplitude is large in the second half of the year with two peaks in one day. The main peak occurs in the daytime, and the duration is longer. The vice peak appears at midnight, and the duration is shorter. The PV power generation is abundant from March to June, deficient in the other months of the year seasonally. The intraday variation is large, but its regularity is similar in each month: The output of PV power increases with the rising of the sun in the morning, and then decreases from the afternoon to the night. Although there are fluctuations in wind power and PV power both in seasonally and daily, they are complementary. In the first half of the year, the output of wind power maintains at a relatively high level, while the output of wind power increases gradually. In the second half of the year, the output of wind power decreases while the output of wind power increases from July to August, and the former output increases while the later output decreases from September to December.

The complementarity of wind and PV power is affected by the installed capacity ratio of wind to PV power. The variation coefficients are calculated by the 1hour and 10-year output of wind/PV power generation. The change of variation coefficients with the installed capacity ratio is shown in Fig.4 (a). With the increase of the installed capacity ratio, the variation coefficient first decreases and then increases, and achieves the minimum variation coefficient of 0.609 when the installed capacity ratio is 0.7 . Therefore, the optimal ratio of installed capacity with the goal of minimizing the output volatility of wind/PV power generation is 0.7 . The cumulative distribution function of the output coefficients of wind/PV power generation with the optimal ratio of installed capacity is shown in Fig.4 (b). Apparently, the maximum output coefficient of wind/PV power with the optimal ratio of installed capacity is 0.672 , and the minimum is 0 . The probability of zero output is $13.15 \%$, implying strong intermittence. 


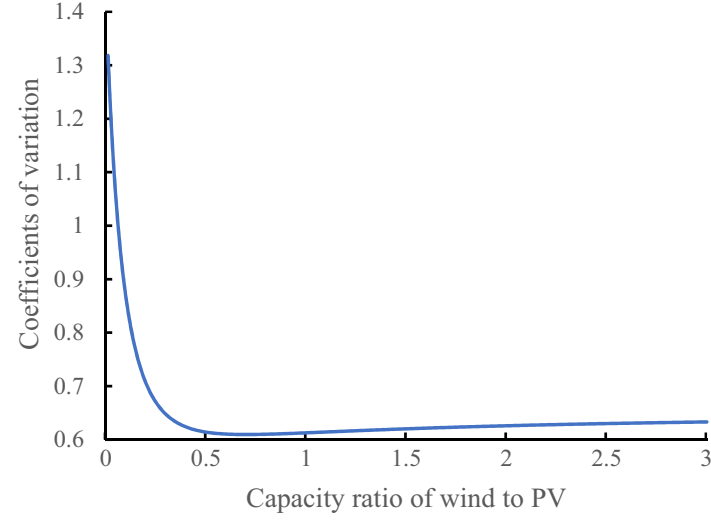

(a)

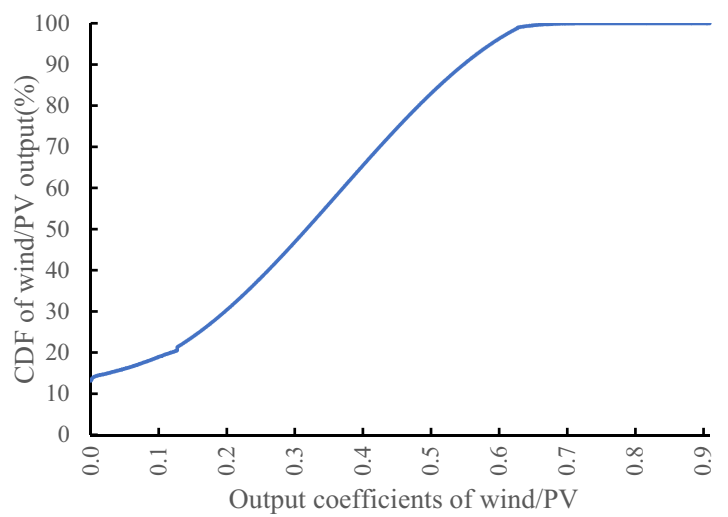

(b)

Fig.4 Relationship between the installed capacity ratio of wind to PV power and the variation coefficients of output of wind/PV joint generation (a). Cumulative distribution function of the output coefficients of wind/PV power with the optimal ratio of installed capacity (b)

\subsection{Complementarity analysis of wind/PV/hydro power}

Based on the optimization model established in this paper, using the data of the year, of which the runoff has a frequency of $50 \%$, the maximum bundled output and the optimal hydropower allocation scheme are obtained. With a $6.3 \mathrm{GW}$ bundled capacity, the maximum bundled output of wind/PV/hydro complementary power generation system is $1.742 \mathrm{GW}$. The optimal hydropower allocation scheme and the grid-connected output of wind/PV power in the dry season are shown in Fig.5. Fig. 5(a) shows the output of different power in the whole dry season, while Fig. 5(b) shows the output in 72-hour. In the figure, filled with the black vertical lines indicates the hydropower output, with the cross lines of the white background indicates the grid-connected output of wind/PV power and with the cross lines of the gray background indicates the abandoned output of wind and PV power.

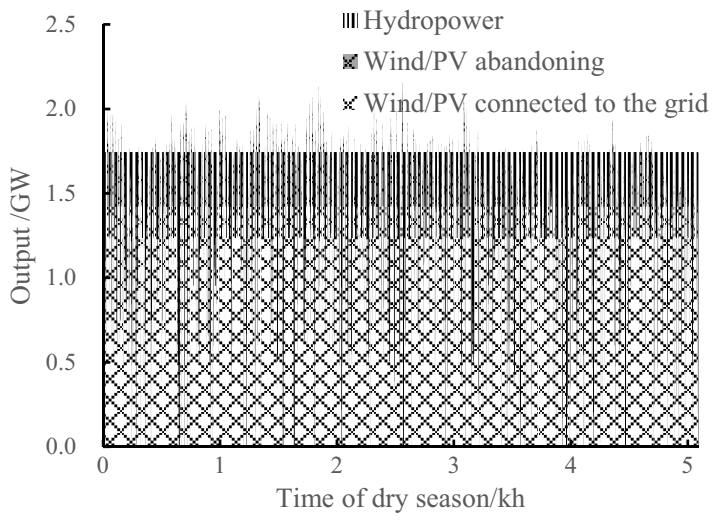

(a)

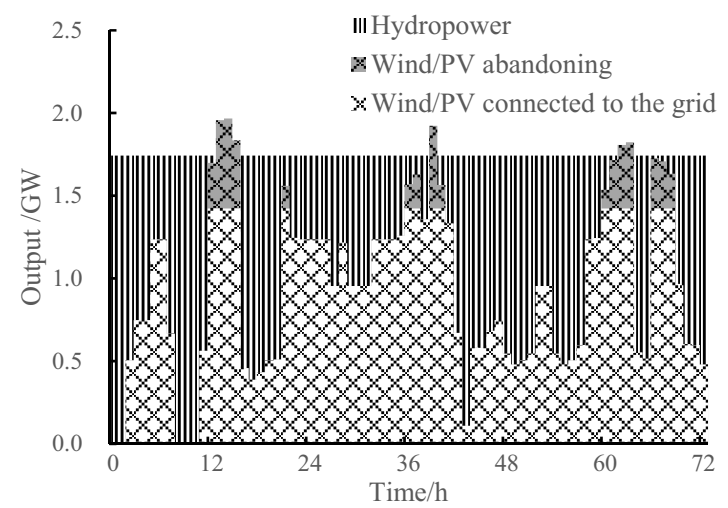

(b)

Fig.5 Output of wind/PV/hydro complementarity system in the dry season

It can be seen from Fig. 5(a) that the wind/PV/hydro complementary power generation system can smoothly output the output of $1.742 \mathrm{GW}$ in each period of the dry season. From Fig. 5 (b), the output of wind/PV power is fluctuant and intermittent, and hydropower plays an important role in stabilizing the output fluctuations of wind/PV power. However, due to the constraints of the minimum output of hydropower and the bundled output of wind/PV/hydro power, the grid-connected output of wind/PV power exceeds the maximum allowable gridconnected output in some periods, such as the 12th to the 15 th, the 60 th to the 63 th, and the 65 th to the 67 th, etc., and the excess part of wind/PV output is discarded.

Categorize 1-hour output of hydropower and wind/PV power generation with the optimal ratio of installed capacity monthly and hourly, respectively. In each month, there are 24 collections, and thus 168 collections for 7 months of the dry season. Fig. 6 shows the variations of the average output of wind/PV power and hydropower in each hour of different months during the dry season. As can be seen, the variation trend of hydropower output is opposite to the trend of wind/PV output. Specifically, the peak of hydropower output and the valley of wind/PV output occur at the same time, which shows great complementary characteristics. 


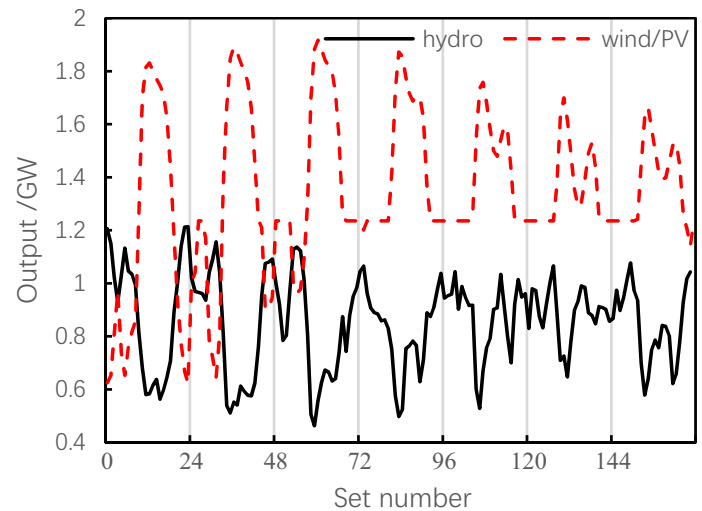

Fig. 6 Variations of average output of wind/PV and hydropower

The complementary characteristics of wind/PV/hydro power are reflected in the ability of hydropower to regulate the wind/PV power to output the power smoothly. During the optimization of the model, it is found that when the model obtains the optimal solution, the constraint (1) reaches the boundary value, that is, the total hydropower generation in the dry season is maximum. Therefore, the ability of hydropower to regulate the output of wind/PV power is limited by the capacity of reservoir and the runoff in the dry season. This regulating ability can be reflected by the bundled output of the complementary system and the absorptive rate of new energy. Higher bundled output and higher absorptive rate implies strong complementary.

Fig. 7 shows the variations of bundled output of wind/PV/hydro power complementary generation system with the bundled installed capacity under two runoff levels. It is worth mentioning that the change of bundled installed capacity of wind/PV/hydro power system is caused by the change of installed capacity of wind and PV power since the hydropower station has been built with determined installed capacity. As can be seen that with the increase of the bundled capacity, the bundled output increases, but the rate of increase becomes slower, indicating that the complementarity among wind, PV and hydro power is getting weaker. The reason is that the difference between the maximum and minimum of joint output of wind/PV power generation increases with the bundled capacity, which calls for higher regulation requirement from the hydropower. However, the regulation ability of hydropower is limited by the installed capacity and runoff. When runoff is larger, a smaller runoff frequency, the regulation ability of hydropower increases, leading to a higher bundled output and a stronger complementarity.

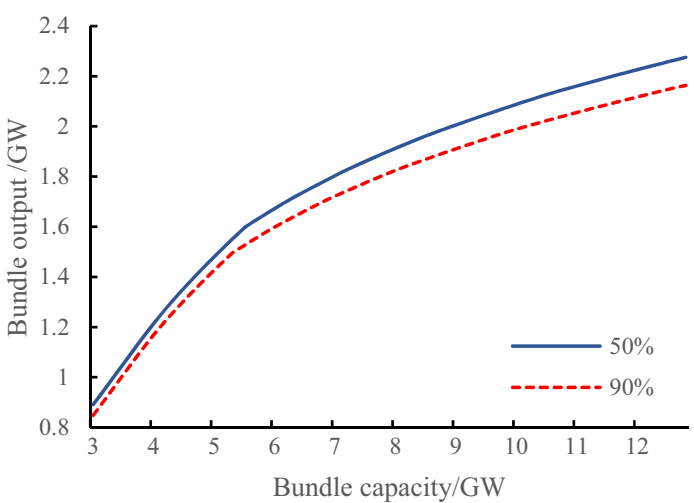

Fig.7. Variation of the bundled output with the bundled installed capacity of the wind/PV/hydro power generation system

In order to compare the complementary between different systems, including the PV/hydro power generation system, wind/hydro power generation system and wind/PV/hydro power generation system, the established model is applied to obtain the absorptive rate of new energy sources, wind or PV. Fig. 8 shows the absorptive rate of new energy sources in three systems under different levels of installed capacity of the new energy. As can be seen, when the new energy installed capacity less than $1.2 \mathrm{GW}$ for the wind/PV/hydro power system, $0.9 \mathrm{GW}$ for the PV/hydro power system, and $1.0 \mathrm{GW}$ for the wind/hydro power system, the new energy absorptive rate is $100 \%$. In other words, the wind and PV power can be absorbed fully by the regulation of hydropower. When the installed capacity of new energy increases beyond the critical value, there exists abandoned energy. With the increase of new energy installed capacity, the new energy absorptive rate of wind/hydro, PV/hydro and wind/PV/hydro power generation complementary system all decrease. Under a given installed capacity of new energy, the absorptive rate for wind/PV/hydro system is largest, implying a highest complementarity. The reason is that the volatility of single power is higher than the complementary new energy systems, and that of wind power is higher than PV power. Higher volatility enhances the difficulty of smoothing power, and decreases the energy absorptive rate.

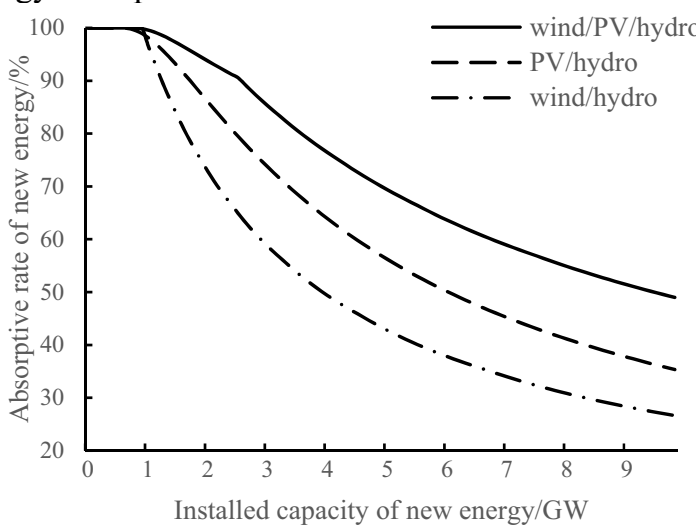

Fig.8. Variations of absorptive rate of new energy with installed capacity under different energy systems

It is worth noting that for the annual and seasonal regulation hydropower stations, hydropower station generates power with installed capacity in flood season and can not smooth the volatility of wind/PV power. 
Therefore, a lot of new energy are abandoned in flood season when there is only hydropower station. If there is other power stations to smooth the volatility of wind/PV power, such as the pumped storage hydropower station, more new energy can be aborted.

\section{Conclusions}

There is complementarity among wind, PV and hydro power, which is closely related to bundled installed capacity of wind/PV/hydro complementary system and regulating ability of hydropower. In this paper, firstly, the ratio of installed capacity of wind to PV power which minimizes the output volatility of wind/PV joint generation is obtained. Secondly an optimization model, with the objective of maximizing grid-connected and steady output of wind/PV/hydro complementary system in the dry season, to determine the bundled output of the system is proposed. Thirdly, the complementary characteristics of wind/PV/hydro power are analyzed by the bundled output and the absorptive rate of new energy in the dry season. The application of this method in the multi-energy system of Yalong River indicates that:

(1)Although both wind and PV power are uncertain, there is complementarity between them. And their complementarity is related to the ratio of installed capacity of wind to PV power. With the ratio of installed capacity increases, the volatility first weakens and then strengthens. The optimal ratio of installed capacity with the goal of minimizing the output volatility of wind/PV power generation is 0.7 . But the probability of zero output is $13.15 \%$, implying strong intermittence.

(2)With a $6.3 \mathrm{GW}$ bundled capacity, the maximum bundled output of wind/PV/hydro complementary power generation system is $1.742 \mathrm{GW}$. With the increase of the bundled capacity, the bundled output increases, but its rate of increase becomes slower, indicating that the complementarity among wind, PV and hydro power is getting weaker. When runoff is larger, the regulation ability of hydropower increases, leading to a higher bundled output and a stronger complementarity.

(3)When the new energy installed capacity less than 1.2GW for the wind/PV/hydro power system, $0.9 \mathrm{GW}$ for the $\mathrm{PV} /$ hydro power system, and $1.0 \mathrm{GW}$ for the wind/hydro power system, the wind and PV power can be absorbed fully. With the increase of the installed capacity of new energy, the new energy absorptive rate of wind/hydro, PV/hydro and wind/PV/hydro complementary power generation system all decrease. Under a given installed capacity of new energy, the new energy absorptive rate for wind/PV/hydro system is largest, implying a highest complementarity.

\section{Acknowledgments}

This study was supported by the National Natural Science Foundation of China (U1765102, 91647201, 51709036).

\section{References}

1. D. Raynaud, B. Hingray, B. François, J.D. Creutin, RENEW ENERG, 125 578-589 (2018).

2. C. Fant, B. Gunturu, A. Schlosser, APPL ENERG, $161565-573$ (2016).

3. Z. Yang, P. Liu, L. Cheng, H. Wang, B. Ming, W. Gong, J CLEAN PROD, 195 562-572 (2018).

4. X. Zhang, G. Ma, W. Huang, S. Chen, S. Zhang, X. Zhang, S. Chen, W. Huang, S. Zhang, G. Ma, 11868 (2018).

5. A. Kies, B.U. Schyska, L. von Bremen, Energy Procedia, 97 149-155 (2016).

6. M. Khan, M.T. Iqbal. RENEW ENERG 30 270282(2005).

7. R.K. Akikur, R. Saidur, H.W. Ping, K.R. Ullah, Renewable and Sustainable Energy Reviews, 27 738-752 (2013).

8. X. Ye, Q. Tang, T. Li, Y.L. Wang, X. Zhang, S.Y. Ye, IOP Conference Series: Materials Science and Engineering, 19912016 (2017).

9. B. François, M. Borga, J.D. Creutin, B. Hingray, D. Raynaud, J.F. Sauterleute, RENEW ENERG, 86 543-553 (2016).

10. G. Notton, S. Diaf, L. Stoyanov. Energy Procedia, 6 574-582 (2011).

11. G. Pianezzola, A. Krenzinger, F.A. Canales. Energy Power Eng. 9 7-15 (2017).

11. R. Billinton, G. Bai. IEEE Transaction on Energy Conversion. 19 574-582 (2004).

12. L. Xu, X. RUAN, B. Zhang. Proceedings of the CSEE. 32 96-106 (2012).

13. G. Bekele, G. Tadesse, APPL ENERG, 97 742-751 (2012). 